\title{
PRESCRIBING TREND AMONG HOSPITALIZED PEDIATRIC PATIENTS IN PRIVATE SECTOR HOSPITAL: A PROSPECTIVE OBSERVATIONAL STUDY IN NADIAD, INDIA
}

\author{
SANDIP B PATEL ${ }^{1 *}$, YASH P PATEL ${ }^{1}$, SHISHAV B SHAH ${ }^{2}$
}

${ }^{1}$ Department of Pharmacology, Ramanbhai Patel College of Pharmacy, Charotar University of Science and Technology, Nadiad, Gujarat, India. ${ }^{2}$ Department of Pediatrics, Shaishav Children Hospital and Neonatal Care Nursery, Nadiad, Gujarat, India. Email: patelsandozrx@gmail.com

Received: 24 January 2020, Revised and Accepted: 18 March 2020

\section{ABSTRACT}

Objectives: Purpose of this study was to analyze the prescription pattern among the hospitalized pediatric patients in private sector hospital.

Methods: The study was conducted from January 2018 to April, 2018 at Shaishav Hospital, Nadiad, Gujarat, India, and 178 patients were included into the study. Results were obtained by applying the World Health Organization (WHO) prescribing indicators as well as Indian Academy of Pediatrics List of Essential Medicines (IAP-LEM).

Results: During the study period, infectious disease such as pneumonitis, bronchitis, enteric fever, gastroenteritis, and dehydration were the dominant disease among the patients. Average number of drugs per prescription was four but in many of prescription, poly-pharmacy has shown. Antibiotic agents were the most prescribed drug (38.6\%) among different classes of drugs. Use of the parenteral route of drug administration was used beyond the range stated by the WHO (59\% prescriptions vs. 13-24\% WHO range). We found that doctor followed IAP-LEM (in $99.78 \%$ cases) for prescribing as well as for dosing also, which was very impressive.

Conclusions: As this study indicates, there is a need of development of evidence-based treatment protocols for common disease condition for the rational use of medication, especially for an antibiotic medication.

Keywords: Drug utilization pattern, Rationale use of medicines, Prescription indicators.

(C) 2020 The Authors. Published by Innovare Academic Sciences Pvt Ltd. This is an open access article under the CC BY license (http://creativecommons. org/licenses/by/4. 0/) DOI: http://dx.doi.org/10.22159/ajpcr.2020.v13i5.36920

\section{INTRODUCTION}

Precise medication could be the key to the achievement of optimal treatment outcomes. Prescription pattern monitoring is an important part of drug utilization processes; prescription pattern monitoring studies (PPMS) is a tool for assessing the prescribing, dispensing, and distribution of medicine. PPMSs are drug utilization studies with the main focus on prescribing, dispensing, and administrating of drugs. They promote the appropriate use of monitored drugs and reduction of abuse or misuse of monitored drugs [1-3].

Prescription pattern analysis is an important parameter of the drug utilization research. Drug utilization research defined by the WHO in 1977 as studies on marketing, distribution, prescription, and use of the drug in society, with special emphasis on the resulting medical, social, and economic consequences [2]. There are some parameters defined by the WHO as the core drug use indicator in "Essential medicine and Health products Information Portal" to study or analyze prescription. The core drug use indicators are divided into (1) prescribing indicators, (2) patient care indicators, and (3) health facility indicators from which prescribing indicators are used to study rationality of the prescriptions.

The infectious disease burden in India is among the highest in the world [4,5]. Infectious diseases are common among pediatric patients in India and contributed to the total mortality rate which is the highest in the world. Pneumonitis and diarrheal disease account for $50 \%$ of a total 1.34 million deaths among Indian children having an age of 1 month-5 years $[6,7]$. Antibiotic agents are among the most commonly prescribed drugs in the pediatric population. Antibiotic resistance, which is a threat to public health, is rapidly increasing globally. The use of antibiotic, including irrational and unnecessary antibiotic treatment, contributes to the development of antibiotic resistance [8]. The rising incidence of bacterial resistance to common antibiotic has made judicious use of antibiotic in pediatric practice a mandate. It has been reported that $20-25 \%$ of antimicrobial usage is questionable or inappropriate [9]. As per the guidelines, prescription medicine should be according to this list of essential medicines. To avoid unnecessary antibiotic prescribing and development of antibiotic resistance, pediatric health-care facilities are advised to follow the IAP-LEM. Therefore, an attempt is made to study the prescription pattern among the hospitalized pediatric population in the private sector hospital of Nadiad, Gujarat, India.

\section{METHODS}

The drug utilization pattern was studied in the pediatric in-patients at a private hospital (Shaishav Children Hospital and Neonatal Care Nursery) located at Nadiad city, Gujarat. The hospital has a total 65 beds, of which 51 are pediatric beds and a 14 neonatal intensive care unit at the time of the study. A prospective observational study was carried out over a period of 4 months (January-April 2018). The study was approved by the human ethics committee of Sat Kaival Hospital Pvt. Ltd., Anand (Study Ref No: SKHPLEC_PROJ/0002). All collected data were anonymized and the need of obtaining written informed consent from the patient was waived. Sat Kaival Hospital Pvt. Ltd., Ethics Committee, Anand, is organized and operates in accordance with the Indian Council of Medical Research Ethical guideline, Good Clinical Practice guideline, and applicable regulations.

All the pediatric patients of either sex from newborn to 21 years of age, prescribed with at least one medication, and had admitted in the pediatric ward for not $<24 \mathrm{~h}$ were included in the study. Outpatients, as well in patients who were admitted $<24 \mathrm{~h}$, were excluded from the study. 
The study excluded prescription items related to blood products, oxygen, total parenteral nutrition, oral rehydration salts, and topical products used during hospitalization, as well as the electrolytes $(0.9 \%$ sodium chloride solution and $5 \%$ glucose solution) of routine use in clinical assistance for the maintenance of peripheral venous access and drug administration.

Demographic details of patients such as age, sex, date of admission, and date of discharge were collected in a case record form. Diagnosis (disease condition) as well drug prescription has been collected from data management software of the hospital. Medications prescribed were recorded in detail such as indications, dosage form, trade/generic name, dose administered, frequency of administration, and route of administration. The antibiotic prescribing data were included in the study of those patients who had at least diagnosed with one of the five most commonly registered infectious disease, i.e. enteric fever, pneumonitis, dehydration, gastroenteritis, and bronchitis.

The data regarding prescription prescribed were analyzed in accordance with prescribing indicators described in "Essential medicine and Health products Information Portal." The complementary parameter such as pediatric classification by age group was done as per the U.S Department of Health and Human Services Food and Drug Administration guideline issued on June 2003. Antibiotic drug classification was done as per the ATC guideline. The comparison of parenteral and oral formulation prescription was done by finding out the percentage formulation (oral as well parenteral) prescribed. Percentage as well as frequency was calculated for different categorical variables, while sum, mean, and range were calculated for continuous variables. All the data were analyzed using Microsoft Office Excel 2016.

\section{RESULTS}

During the study period of 4 months (January 2018-April 2018) total, 178 patients were included into the study; classification of a pediatric patient as per the age group as well as gender distribution has been done as per the United States Food and Drug Administration guideline (Table 1).

Mean duration of admission was 3.70 days. The duration of hospital stay was a bit higher in female patients (3.81 days) compare to male patients (3.64).

Gastrointestinal infections accounted for the highest number of cases (62.83\%), including dehydration (40.47\%), enteric fever $(7.61 \%)$, enteritis $(14.28 \%)$, and hepatitis $(0.47 \%)$. Disease condition such as pneumonitis $(16.20 \%)$ and bronchitis $(16.60 \%)$ also had a large number of cases. Patients were diagnosed with other severe lifethreatening conditions such as swine flu and dengue (Table 2).

From 178 different prescriptions, a total of 648 drugs were prescribed in all the cases with 59\% parenteral and $41 \%$ oral formulation for different disease conditions. Pharmacological classification of the drugs has been reported in Table 3

Average number of drug per prescription is use to check polypharmacy. The mean drugs prescribed per prescription was 4 though, 47 prescriptions (26\%) had prescribed 5 or 6 drugs per prescription. During 4 months of the study period, not a single prescription was prescribed with generic names. All the drugs were prescribed by

Table 1: Classification of pediatrics and gender distribution

\begin{tabular}{|c|c|c|c|}
\hline \multirow[t]{2}{*}{ Age group } & \multirow{2}{*}{$\begin{array}{l}\text { Number of } \\
\text { patients } \\
(n=178)(\%)\end{array}$} & \multicolumn{2}{|c|}{ Gender distribution (\%) } \\
\hline & & Male (n=109) & Female $(n=69)$ \\
\hline New bor & $6(3)$ & $2(33)$ & $4(67)$ \\
\hline Infants & $46(26)$ & $30(65)$ & $16(35)$ \\
\hline Child & 119 (67) & $72(60)$ & $47(40)$ \\
\hline Adolescents & $7(4)$ & $5(71)$ & $2(29)$ \\
\hline
\end{tabular}

trade name. Almost all the prescription had prescribed with at least one antibiotic medication from different pharmacological class. A total of 250 antibiotics was prescribed. Among them, amikacin (aminoglycoside) was accounted for $34 \%$ of total prescribing. All the prescriptions were prescribed with at least one parenteral formulation. We found that all the medicines prescribed were from the essential medicine list.

Ceftriaxone, a systemic antibiotic, has the highest number of prescriptions $(103,15.89 \%)$ followed by another systemic antibiotic drug amikacin $(87,13.42 \%)$ (Table 4).

Fixed-dose combinations (FDCs) are one of the strategies to reduce the number of drugs prescribed and improve medication compliance. Increasing the requirement of drugs in patients with more than one disease justifies the use of FDCs. During the study period total, 580 different formulations were prescribed, from which 169 (29\%) were the FDC.

Table 2: Distribution of disease conditions

\begin{tabular}{|c|c|c|}
\hline Infection type (\%) & $\begin{array}{l}\text { Disease } \\
\text { conditions }(n=210)(\%)\end{array}$ & $\begin{array}{l}\text { Avg. Duration } \\
\text { of hospital stay } \\
\text { (days) }\end{array}$ \\
\hline Respiratory & Pneumonitis $(n=34,16.20)$ & 4.5 \\
\hline \multirow{2}{*}{ infection (33.27) } & $16.60)$ & 3 \\
\hline & Swine flu ( $\mathrm{n}=1,0.47)$ & 7 \\
\hline \multirow{4}{*}{$\begin{array}{l}\text { Gastrointestinal } \\
\text { infection } \\
(62.83)\end{array}$} & Dehydration $(\mathrm{n}=85,40.47)$ & 3.6 \\
\hline & Enteric fever $(n=16,7.61)$ & 3.5 \\
\hline & Enteritis $(n=30,14.28)$ & 3 \\
\hline & Hepatitis ( $\mathrm{n}=1,0.47)$ & 5 \\
\hline CNS disorders (0.47) & Encephalopathy $(\mathrm{n}=1,0.47)$ & 5 \\
\hline \multirow[t]{2}{*}{ Viral infection (3.43) } & Viral fever $(n=3,1.43)$ & 3 \\
\hline & Dengue $(n=4,2.0)$ & 4 \\
\hline
\end{tabular}

Table 3: Classification of medicines

\begin{tabular}{ll}
\hline Medicine class & $\begin{array}{l}\text { Number of } \\
\text { prescriptions (n=648) }\end{array}$ \\
\hline Anti-infective for systemic use (J) & $255(39.37)$ \\
Antiviral agent (J05) & $2(0.31)$ \\
Antibiotic agents (J01) & $250(38.6)$ \\
Antifungal agents (J02) & $3(0.46)$ \\
Respiratory system (R) & $105(16.2)$ \\
Antiasthmatics for systemic use (R06) & $12(1.85)$ \\
Antiallergics (R06) & $43(6.64)$ \\
Cold and cough preparation (R05) & $50(7.71)$ \\
Musculoskeletal system (M) & $84(12.96)$ \\
NSAIDs (M01) & $84(12.96)$ \\
Alimentary tract and metabolism (A) & $103(15.89)$ \\
Laxatives (A06) & $8(1.23)$ \\
Antiemetics (A04) & $38(5.86)$ \\
Antiulcer agents (A02) & $57(8.80)$ \\
Dermatological (D) & $6(0.92)$ \\
Corticosteroids (D07) & $6(0.92)$ \\
Vitamin supplements (V06) & $95(14.66)$ \\
\hline
\end{tabular}

Table 4: Most frequently prescribed drugs

\begin{tabular}{ll}
\hline Drug & Prescriptions (n=648, 100\%) \\
\hline Ceftriaxone & $103(15.89)$ \\
Amikacin & $87(13.42)$ \\
Paracetamol & $65(10.03)$ \\
Ranitidine & $53(8.18)$ \\
Ondansetron & $38(5.86)$ \\
Ambroxol & $24(3.70)$ \\
Chlorpheniramine & $27(4.17)$ \\
Sodium citrate & $19(2.93)$ \\
Ibuprofen & $16(2.47)$ \\
Dextromethorphan & $11(1.70)$ \\
\hline
\end{tabular}




\section{DISCUSSION}

It is evident from the many studies that evidence-based guidelines do not follow in more than one-third of antimicrobial treatments in adults. Similar or worse results in pediatrics are expectable since lack of pharmacokinetic and pharmacodynamics studies hinder the establishment of optimal dosing and intervals for antimicrobial prescription adjusted to different age groups. Further, in pediatrics, antimicrobial prescriptions are more common to provide treatment of infection which is not focused on searching for the causative microorganisms, resulting empirical treatment of "suspected infection" or "risk of infection" based in age group and patient's symptoms. Empirical treatment increases the chances of therapeutic failure and promotes the use of antibiotic with a broader spectrum which may not be required for the treatment of infectious diseases $[8,9]$.

The present investigation was carried out to provide insight related to prescription pattern among hospitalized pediatric patients in a private hospital of Nadiad, Gujarat. Of 178 patients, most of the patients were male $(n=109 ; 61 \%)$ followed by female $(n=69 ; 39 \%)$. Our results were similar to the studies conducted by Pashankar and Loening-Baucke (male patients 62\% and female patients 38\%) [10] and Karande et al. (male 59.8\% and female patients $40.2 \%$ ) [11]. Our study results state that most of the patients were prescribed with 59\% intravenous injections; $34 \%$ syrup/oral drops/nasal drops and $7 \%$ as tablets/ capsules whereas, in Thiruthopu et al. study, $63 \%$ of medicines were prescribed as intravenous injection, $15 \%$ syrup/oral drops/nasal drops, $17 \%$ were prescribed as tablets/capsules, and others were $4.1 \%$ [12].

In the present study, on an average four medicines were prescribed per patient, which is much higher as compared to 1.4 and 2.3 in similar studies from Sanz and Boada, Walter et al., and Mirza et al. showed the average number of medicines to be 3.3 per prescription in adult patients [13-15]. Pandey et al. and Ansari etal. found this number to be 5.9 and 5.1 medicines per prescription, respectively, in their studies $[16,17]$. Thus, it is evident that the polypharmacy and overprescribing are common in India, an economically developing country, as compared to economically developed western countries. In our study, we observed extensive use of antibiotics. The number of encounters with antibiotics was $250(37.9 \%)$ followed by non-steroidal anti-inflammatory medicines $84(12.7 \%)$ and antiulcer class of medicines 57 (8.66\%). Third-generation cephalosporins were the most commonly prescribed antibiotic group. The patients with enteric fever, gastroenteritis, and respiratory tract infections (RTI) were commonly prescribed thirdgeneration cephalosporins mostly through the parenteral route. The similar observations were found in the study done by Sharma et al. [18] According to the WHO, broad-spectrum antibiotics are recommended only when treatment with specific antibiotics has been proven ineffective.

FDCs were more commonly prescribed, especially to patients with acute gastroenteritis, RTI, and enteric fever. The FDCs are usually costlier than single drug formulation; thus, an extra cost of treatment is directly imposed on the patients. In almost all patients in study settings, the antibiotics were prescribed empirically but without any laboratory diagnostics; therefore, the rationality of the prescribed FDCs could not be evaluated. The probable unnecessary exposure to antibiotics for viral infections and broad-spectrum antibiotics, including FDCs, could potentially lead to the development of antibiotic resistance [7]. This study showed that most of the indicators analyzed were in agreement with the WHO's recommendations, except for the mean number of drugs per encounter and percentage of medications prescribed by generic name which should be $\leq 2$ and $100 \%$, respectively.

Prescribing generic name is known to reduce the cost of drug treatment and rationalizing drug therapy. In this study, none of the prescriptions contain a single drug prescribed in its generic name. This varies from $13.3 \%$ to $93 \%$ across the globe. A similar study conducted in Goa, the western part of India, has similar observations where only $0.05 \%$ of the drugs of 1842 products prescribed were in the generic name [19]. The results of work conducted in India report this as 3.8\%. One of the reasons for poor prescribing by generic name is the non-availability of the pediatric formulations in the hospital pharmacy. Prescribing of antibiotics does not fell within the WHO recommended range of 20$26 \%$, but it was higher $(37.9 \%)$ than the range recommended while prescribing of injections was beyond the recommended range of the WHO (51\% vs. $13.4-24.0 \%)$. A higher rate of injections was prescribed in other Indian studies [12]. The results show that prescribing of essential medicines from the IAP-LEM was higher (99.78\%) at a hospital which was a higher percentage than studies of Sharma et al. [7] Earlier studies have shown a reduction of broad-spectrum antibiotic use in health-care facilities after implementation of local prescribing guidelines which can reduce irrational antibiotic prescribing. In the study, a higher proportion of antibiotics (56\%) were present from the list of essential antibiotics [20].

\section{CONCLUSIONS}

This study highlights the prescription pattern of different medicines in different age groups in hospitalized pediatric populations. We came to know that almost all the prescriptions have prescribed with at least one antibiotic medicine. It highlights the need of diagnosis-specific antibiotic prescription as well the development of a better system which can regulate the use of antibiotics in pediatric populations as it could be the major reason for antibiotic resistance in the future. All the medications were prescribed by brand name rather than generic names. Our study has been shown that almost all the drugs were prescribed as per the standard treatment guideline for pediatric population in India, suggesting compliance with the IAP-LEM guidelines for the selection of the medicines.

\section{AUTHORS' CONTRIBUTIONS}

Dr. Sandip Patel: Concept, Statistical analysis, Manuscript Review; Mr. Yash Patel: Literature search, data collection, and manuscript preparation; Dr. Shaishav Shah: Data collection and manuscript review.

\section{CONFLICTS OF INTEREST}

The authors declared no conflicts of interest.

\section{AUTHORS' FUNDING}

Ramanbhai Patel College of Pharmacy.

\section{REFERENCES}

1. Jain S, Upadhyaya P, Goyal J, Kumar A, Jain P, Seth V, et al. A systematic review of prescription pattern monitoring studies and their effectiveness in promoting rational use of medicines. Perspect Clin Res 2015;6:86-90.

2. World Health Organisation. Introduction to Drug Utilization Research. Geneva: World Health Organisation; 2003.

3. Errors M. Technical Series on Safer Primary Care. Geneva: World Health Organization; 2016.

4. Kumar SG, Adithan C, Harish BN, Sujatha S, Roy G, Malini A. Antimicrobial resistance in India: A review. J Nat Sci Boil Med 2013;4:286-91.

5. Global Antibiotic Resistance Partnership (GARP)-India Working Group. Rationalizing antibiotic use to limit antibiotic resistance in India. Indian J Med Res 2011;134:281-94.

6. Karimi A, Haerizadeh M, Soleymani F, Haerizadeh M, Taheri F. Evaluation of medicine prescription pattern using World Health Organization prescribing indicators in Iran: A cross-sectional study. J Res Pharm Prac 2014;3:39-45.

7. Sharma M, Damlin A, Pathak A, Lundborg CS. Antibiotic prescribing among pediatric inpatients with potential infections in two private sector hospitals in central India. PLoS One 2015;10:e0142317.

8. World Health Organization. The Evolving Threat of Antimicrobial Resistance: Options for Action. Geneva: World Health Organization; 2012.

9. Kotwani A, Holloway K. Trends in antibiotic use among outpatients in New Delhi, India. BMC Infect Dis 2011;11:99.

10. Pashankar DS, Loening-Baucke V. Increased prevalence of obesity in children with functional constipation evaluated in an academic medical center. Pediatrics 2005;116:e377-80. 
11. Karande S, Sankhe P, Kulkarni M. Patterns of prescription and drug dispensing. Indian J Pediatr 2005;72:117-21.

12. Thiruthopu NS, Mateti UV, Bairi R, Sivva D, Martha S. Drug utilization pattern in South Indian pediatric population: A prospective study. Perspect Clin Res 2014;5:178-83.

13. Sanz EJ, Boada JN. Drug utilization by children in Tenerife Island. Eur J Clin Pharmacol 1988;34:495-99.

14. Walter AW, Mulhern RK, Gajjar A, Heideman RL, Reardon D, Sanford RA, et al. Survival and neurodevelopmental outcome of young children with medulloblastoma at St Jude children's research hospital. J Clin Oncol 1999;17:3720-8.

15. Mirza NY, Desai S, Ganguly B. Prescribing pattern in a pediatric outpatient department in Gujarat. Bangladesh J Pharmacol 2009;4:39-42.

16. Pandey AA, Thakre SB, Bhatkule PR. Prescription analysis of pediatric outpatient practice in Nagpur city. Indian J Community Med
2010;35:70-3.

17. Ansari F, Gray K, Nathwani D, Phillips G, Ogston S, Ramsay C, et al. Outcomes of an intervention to improve hospital antibiotic prescribing: Interrupted time series with segmented regression analysis. J Antimicrob Chemother 2003;52:842-8.

18. Sharma M, Eriksson B, Marrone G, Dhaneria S, Lundborg CS. Antibiotic prescribing in two private sector hospitals; one teaching and one non-teaching: A cross-sectional study in Ujjain, India. BMC Infect Dis $2012 ; 12: 155$

19. Patel V, Vaidya R, Naik D, Borker P. Irrational drug use in India: A prescription survey from Goa. J Postgrad Med 2005;51:9-12.

20. Pathak D, Pathak A, Marrone G, Diwan V, Lundborg CS. Adherence to treatment guidelines for acute diarrhoea in children up to 12 years in Ujjain, India-a cross-sectional prescription analysis. BMC Infect Dis 2011;11:32. 